\title{
Planarizable Supersymmetric Quantum Toboggans ${ }^{\star}$
}

\author{
Miloslav ZNOJIL
}

Nuclear Physics Institute ASCR, 25068 Řez, Czech Republic

E-mail: znojil@ujf.cas.cz

URL: http://gemma.ujf.cas.cz/ znojil/

Received November 30, 2010, in final form February 21, 2011; Published online February 25, 2011

doi:10.3842/SIGMA.2011.018

\begin{abstract}
In supersymmetric quantum mechanics the emergence of a singularity may lead to the breakdown of isospectrality between partner potentials. One of the regularization recipes is based on a topologically nontrivial, multisheeted complex deformations of the line of coordinate $x$ giving the so called quantum toboggan models (QTM). The consistent theoretical background of this recipe is briefly reviewed. Then, certain supersymmetric QTM pairs are shown exceptional and reducible to doublets of non-singular ordinary differential equations a.k.a. Sturm-Schrödinger equations containing a weighted energy $E \rightarrow E W(x)$ and living in single complex plane.
\end{abstract}

Key words: supersymmetry; Schrödinger equation; complexified coordinates; changes of variables; single-complex-plane images of Riemann surfaces

2010 Mathematics Subject Classification: 81Q60; 81Q12; 46C15; 81Q10; 34L20; 47A75; $47 \mathrm{~B} 50$

\section{Introduction}

Within the framework of the standard supersymmetric quantum mechanics (SUSY QM as reviewed, e.g., by Cooper et al. [1]) the interaction potentials $V(x)$ must be assumed regular. Otherwise, in a way pointed out by Jevicki and Rodrigues [2] the usual correspondence between spectra of the SUSY-related "partner" Hamiltonians might disappear. The SUSY-induced parallels between the bound states generated by the partner potentials would break down [3]. The main phenomenological merit of the formalism would be lost. In the language of mathematics, with the singularity emerging at least in one of the partner potentials, even the respective Hilbert spaces may become different. For illustration of details one may consult, e.g., Fig. 12.1 of [1] or the explicit construction of an example by Das and Pernice [4].

In the spirit of the $\mathcal{P} \mathcal{T}$-symmetric quantum mechanics (PTS QM, introduced and made popular mainly in $[5,6,7,8,9,10])$ we returned to the Jevicki's and Rodrigues' problem. In a comment [11] on [4] we recommended the regularization of the strong singularities in $V(x)$ mediated by a suitable small complex deformation of the line of coordinates. The requirement of the smallness of the $\mathcal{P} \mathcal{T}$-symmetric deformation $\mathcal{C} \rightarrow \mathbb{R}$ of the line of coordinates appeared essential as long as the regularized Hamiltonians themselves became manifestly non-Hermitian, $H \neq H^{\dagger}$.

During the subsequent development of the theory a full understanding has been achieved of the formal foundations of the recipe based on the use of non-Hermitian operators of observables with real spectra. The recent reviews $[12,13,14,15]$ my be recalled as confirming the full compatibility of $\mathcal{P} \mathcal{T}$-symmetric models with the standard postulates of quantum mechanics.

The resulting extension of the practical scope of quantum theory opened the path towards the proposal of a new class of regularization recipes [16, 17]. They may be characterized by the

${ }^{\star}$ This paper is a contribution to the Proceedings of the Workshop "Supersymmetric Quantum Mechanics and Spectral Design" (July 18-30, 2010, Benasque, Spain). The full collection is available at http://www.emis.de/journals/SIGMA/SUSYQM2010.html 
use of arbitrarily deformed and even topologically nontrivial complex curves $x=x(s) \in \mathcal{C} \subset \mathbb{C}$ of generalized coordinates defined over certain topologically nontrivial Riemann surfaces $\mathcal{R}$.

In a number of concrete non-SUSY examples [18], the latter curves $\mathcal{C}$ were, typically, chosen as spiralling around the branch points of the wave functions $\psi(x)$. For this reason we coined the name of "quantum toboggan models" (QTM) for all of the models supported by a topologically nontrivial (i.e., multisheeted) integration curve $\mathcal{C}$ along which the latter wave functions $\psi(x)$ are, by construction, analytic.

In our most recent paper on the QTM [19] we returned to [11] and indicated that the menu of possible QTM regularizations of SUSY may be broader than expected. New and surprising connections between algebraic (SUSY) and analytic properties of wave functions were recently revealed [20]. In this context the use of the regularizations leads again to a number of new open questions (cf., e.g., [21]). For this reason we are also returning to the subject in our present paper.

In addition to an overall outline of the relevant parts of the theory, we plan to present here also a few new and unpublished results. First, in Section 2 a compact summary will be given of the traditional role of SUSY in quantum mechanics. Second, Section 3 will review briefly the introduction and a few relevant results obtained during the most recent QTM constructions. Next, Sections 4 and 5 will offer a thorough explanation of compatibility between QTM constructions and the standard probabilistic interpretation and postulates of quantum mechanics. The key ingredients in these explanations will be the simplification of the QTM mathematics via a "rectification" change of variables and the subsequent construction of the "standard" Hilbert space $\mathcal{H}^{(S)}$ by means of the introduction of certain not entirely standard inner products.

A few open problems will finally be addressed in Section 6 (including also our main illustrative example of planarization) and in a few appendices. Thus, in Appendix A we shall explain that the non-Hermiticity property $H \neq H^{\dagger}$ of the regularized Hamiltonians (say, of [11]) is just an artifact of an inadequate choice of the "false" Hilbert space $\mathcal{H}^{(F)}$ where the superscript may also hint that this space is only "too friendly" (cf. also [15] for additional details). In subsequent Appendix B we shall make use of this theoretical background attaching three Hilbert spaces to the single quantum system. In Appendix $\mathrm{C}$ we shall describe a more concrete implementation of the resulting innovative "three-Hilbert-space" quantum mechanics (THS QM) to the tobogganic version of popular $V(x)=i x^{3}$. Finally, all these preliminary considerations will be fructified after return to Section 6 where a synthesis of the idea of SUSY with the idea of $\mathcal{P} \mathcal{T}$-symmetry will be presented (and summarized in Section 7).

\section{Supersymmetric Schrödinger equations}

Let us recall that, paradoxically, the recent intensive development of the mathematics of SUSY QM failed to reach its original goals (in particle physics) but still may be considered very successful, especially in its role of a tool for study of mutual relations between different potentials in the quantum bound state problem.

The basic idea of SYSY QM is virtually elementary: in terms of the two differential operators

$$
A=-\frac{d}{d x}+\mathcal{W}(x), \quad B=\frac{d}{d x}+\mathcal{W}(x)
$$

defined in terms of the calligraphic-font "superpotential" $\mathcal{W}(x)$ one constructs the calligraphicfont partitioned Hamiltonian

$$
\mathcal{H}=\left[\begin{array}{cc}
H^{(U)} & 0 \\
0 & H^{(L)}
\end{array}\right]=\left[\begin{array}{cc}
B A & 0 \\
0 & A B
\end{array}\right],
$$

where the superscripts $(U, L)$ stand for the "upper" and "lower" partition, respectively. The other two calligraphic-font partitioned operators are also introduced as playing the role of the 
supercharges,

$$
\mathcal{Q}=\left[\begin{array}{ll}
0 & 0 \\
A & 0
\end{array}\right], \quad \tilde{\mathcal{Q}}=\left[\begin{array}{ll}
0 & B \\
0 & 0
\end{array}\right] .
$$

These operators are easily shown to generate one of the simplest SUSY algebras (cf. [1] for all the details and multiple consequences).

In the spirit of our comment [11] it is important to consider operators (1) without any additional assumption requiring the reality and regularity of the superpotentials $\mathcal{W}(x)$. As a consequence, operators $A$ and $B$ need not necessarily play the role of the usual annihilation and creation operators, etc. For the first time we used this innovative flexibility of superpotentials $\mathcal{W}(x)$ in [22] (cf. also [23]) where we managed to regularize

$$
V \sim \frac{1}{r^{2}} \Longrightarrow V \sim \frac{1}{(t-i \varepsilon)^{2}}, \quad r \in \mathbb{R}^{+}, \quad t \in \mathbb{R}
$$

via an additional redefinition

$$
A=-\mathcal{T} \frac{d}{d x}+\mathcal{T} \mathcal{W}(x), \quad B=\frac{d}{d x} \mathcal{T}+\mathcal{W}(x) \mathcal{T}
$$

The choice of the new auxiliary involution operator $\mathcal{T}$ remained unrestricted by the algebraic considerations.

In the above-mentioned amendment of the formalism [19] we merely relaxed the involutivity property $\mathcal{T}^{2}=I$ and postulated

$$
A=-\mathcal{T} \frac{d}{d x}+\mathcal{T} \mathcal{W}(x), \quad B=\frac{d}{d x} \mathcal{T}^{-1}+\mathcal{W}(x) \mathcal{T}^{-1} .
$$

Now we intend to develop this idea further on.

The first technical ingredient needed for a consistent build up of the SUSY version of the theory lies in the necessary clarification of the change of the role of the Hermitian conjugation operation during and after the supersymmetrization. The discussion of this point will be given below. Here, in introduction, we would only like to remind the readers that the use of the complex superpotentials $\mathcal{W}=-\psi_{0}^{\prime} / \psi_{0}$ proved extremely productive, especially by leading to many new solvable Schrödinger equations (cf. $[24,25])$ in which the authors managed to circumvent the obstructions connected with the "seed" wave functions $\psi_{0}$ having nodal zeros.

\section{Tobogganic Schrödinger equations}

Even the earliest papers on the models described by equation (8) of Appendix A [26] already reported the emergence of the real bound-state energies for certain non-real potentials $V(x)$ and/or for certain non-real paths of integration $\mathcal{C}(s) \neq \mathbb{R}$ of the Schrödinger equation. These papers found their motivation in perturbation theory and their authors did not pretend to offer any truly physical predictions. The situation has changed after the publication of the influential letter [6]. The subsequent massive return of interest to the apparently non-Hermitian models with real spectra resulted in the discoveries of many new exactly solvable analytic models [27], square-well-shaped models [28], point interaction models [29], Calogero-type many-body models [30] and, last but not least, of the tobogganic models in which the paths $\mathcal{C}(s)$ were chosen as spiralling around the branch points of their (by assumption, analytic) wave functions $\psi(x)$.

Teaching by example let us recall here the most elementary (viz., force-free, radial, purely kinematical) Hamiltonian $H$ of [31],

$$
H=-\frac{d^{2}}{d x^{2}}+\frac{\ell(\ell+1)}{x^{2}}, \quad \ell \in \mathbb{R} .
$$




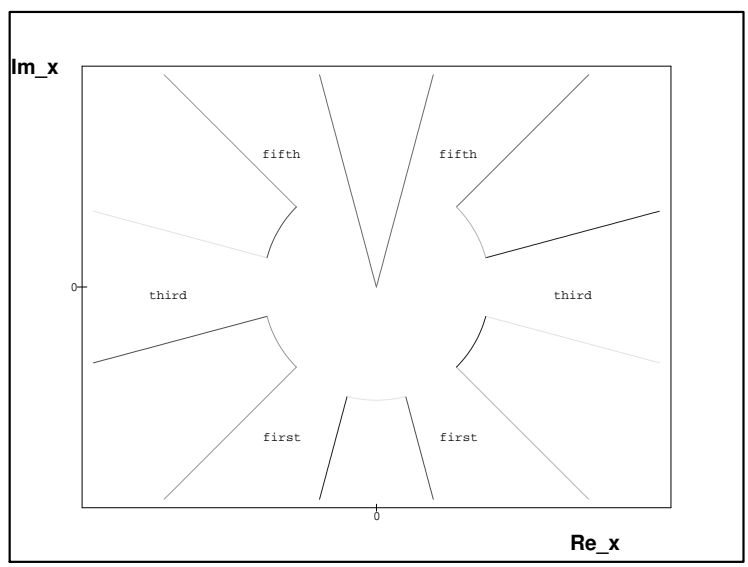

Figure 1. The allowed wedges for the non-tobogganic, single-complex-plane integration contours for potential $V(x)=x^{2}(i x)^{8}$ and for the preselected wave-function asymptotics $\psi(x) \sim \exp -x^{6} / 6$.

In a small complex vicinity of the origin any related wave function $\psi(x)$ has, obviously, the well known form

$$
\psi(x)=c_{+} \psi^{(+)}(x)+c_{-} \psi^{(-)}(x),
$$

where

$$
\psi^{(+)}(x)=x^{\ell+1}+\text { corrections, } \quad \psi^{(-)}(x)=x^{-\ell}+\text { corrections, } \quad|x| \ll 1 .
$$

This means that at a generic real $\ell>-1 / 2$ the corresponding Riemann surface $\mathcal{R}$ will posses a branch point at $x=0$. Whenever this value of $\ell$ becomes rational, the corresponding Riemann surface $\mathcal{R}$ will be glued of a finite number of separate sheets. Obviously, the study of the analytic properties of wave functions only becomes sufficiently well known at the integer values of $\ell$ (cf. also a comment on this point in [31]).

\subsection{The single-Riemann-sheet (i.e., cut-plane) models}

The most natural way out of the difficulties which characterize the general choice of $\ell$ lies, obviously, in the simplest special choice of $\ell=0$. Even then, the study of the most popular complex potentials $V(q)=(i q)^{\delta} q^{2}$ requires a number of technical simplifications which lead, according to the recommendations in [6], to the most stable results when the path $\mathcal{C}(s)$ remains confined to a single cut complex plane. At any $\delta \geq 0$ one can then define an "optimal" set of curves (i.e., $\delta$-dependent asymptotic boundary conditions) for which all of the bound-state energy levels $E_{n}$ behave as smooth functions of the couplings $\lambda$ or of the other variable parameters collected in a suitable multiindex $\vec{\lambda}$ entering the energies as their argument, $E_{n}=E_{n}(\vec{\lambda})$.

For illustration we may pick up, say, the asymptotically decadic potential $V(x)=\lambda(i x)^{8} x^{2}$ and declare, say, the specific asymptotics $\psi(x) \sim \exp \left(-x^{6} / 6\right)$ of wave functions physical at $|x| \gg 1$. Then, the coupling-independent triplet of the allowed left-right-symmetric asymptotical wedges exists in a way hinted by Fig. 1 where we did set $\lambda=1$ without loss of generality.

Naturally, beyond the single complex plane (with an upwards-oriented cut) the heuristically extremely successful left-right symmetry of curves $\mathcal{C}(s)$ might be also preserved - this was the basic idea of the introduction of quantum toboggans in [17].

\subsection{The more-Riemann-sheets models (quantum toboggans)}

Let us assume that our wave functions $\psi(x)$ possess just a single branch-point singularity (located in the origin) and that a family of tobogganic curves $\mathcal{C}^{(\alpha)}(s)$ is defined as the set of paths over 


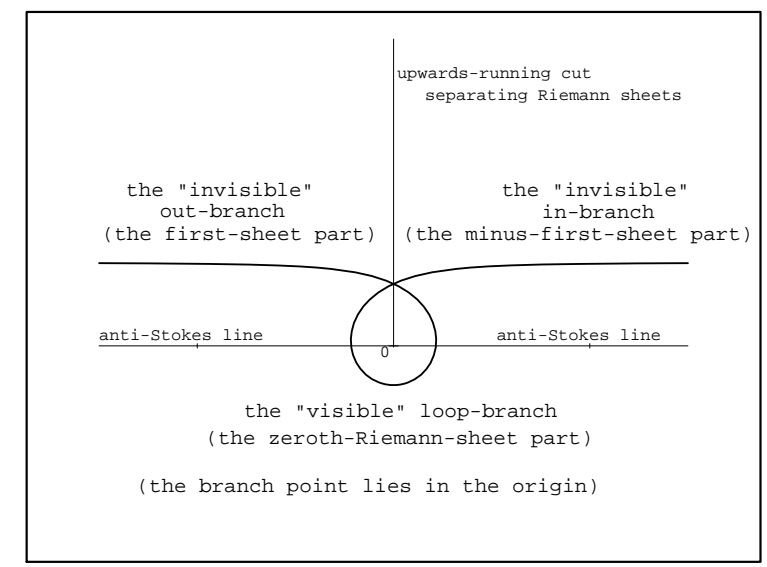

Figure 2. An example of a single-branch tobogganic curve $\mathcal{C}^{(\pi)}$.

the Riemann surface $\mathcal{R}$ which are left-right symmetric in projection to the cut complex plane. Moreover, we shall assume that these curves remain

1) locally linear and parallelling the real axis at $|s| \ll 1$; this means that they may be then prescribed by the formula $\mathcal{C}^{(\alpha)}(s)=-\mathrm{i} \varepsilon+s+\mathcal{O}\left(s^{2}\right)$ at a suitable shift-parameter $\varepsilon>0$;

2 ) turning upwards by the angle $\alpha>0$ during the growth of $s \in(0, \infty)$ while disappearing "behind the cut" (i.e., to the first Riemann sheet, etc.), provided only that $\alpha>\pi / 2$;

3) turning upwards by the angle $\alpha>0$ during the growth of $t=-s \in(0, \infty)$ while disappearing "behind the cut" (i.e., to the minus-first Riemann sheet, etc.), provided only that $\alpha>\pi / 2$;

4) encircling the branch point by the total angle $2 \alpha$ and becoming again approximately linear at large $|s| \gg 1$ (cf. the illustrative sample of $\mathcal{C}^{(\alpha)}$ with $\alpha=\pi$ given in Fig. 2).

At the present stage of development of the general theory of quantum toboggans [32] our insight in their structure based on the existing purely numerical studies of their spectra cannot still be considered satisfactory. In particular, the bad news were communicated by Gerd Wessels [33] who merely managed to show how the standard numerical methods of Runge-Kutta class succeed in some of the most standard non-tobogganic models (8). His numerical algorithms failed to converge in the domains of the expected reality of the tobogganic spectra. Similar incomplete preliminary calculations were also reported by Hynek Bíla [34].

Fortunately, our recent attempted application of perturbation theory (briefly summarized in Appendix $\mathrm{C}$ below) succeeded in the quantitative estimate of the topology-dependence of the tobogganic stable-bound-state spectra in the large- $\ell$ dynamical regime. The calculations have already been performed for the most important special case of the well known toy model where $V(x) \sim \mathrm{i} x^{3}$. The current $1 / \ell$ expansion technique proved applicable and consistent. Our calculations led to the prediction of the nontrivial leading-order topology-dependence of the low-lying energy levels.

These conclusions cannot be directly extended to the domain of small $\ell$ where the topologydependence of the tobogganic stable-bound-state spectra may be expected different. In particular, it may prove much more sensitive to the details of the behavior of the wave functions near the branch point. This expectation may be extracted from the interesting non-tobogganic numerical study [35]. We believe that a new insight in this technical subtlety might also prove obtainable after the incorporation of the very efficient machinery of SUSY QM in combination with the new set of tricks available within the PTS QM framework. In particular, solvable examples should be mentioned as an important tool in the QTM analysis. 


\subsection{A quantum-toboggan example which is exactly solvable}

Theorem 1 (cf. [31]). The tobogganic Schrödinger equation

$$
-\frac{d^{2}}{d r^{2}} \psi(r)+\frac{\ell(\ell+1)}{r^{2}} \psi(r)=E \psi(r), \quad \ell=n+\frac{D-3}{2}
$$

which is perturbed by the short-range potential $V(r)=\gamma / r^{2}$ of a quantized strength

$$
\gamma=\left(\frac{M}{2 N}\right)^{2}-\left(n+\frac{D-2}{2}\right)^{2}
$$

possesses a closed-form bound-state solution $\psi(r) \in \mathbb{L}^{2}\left(\mathcal{C}^{(\alpha)}\right)$ at any integer spatial dimension parameter $D$, integer angular momentum parameter $n$, integer winding number $N=\alpha / \pi$ and an "allowed" integer $M=1,2,3, \ldots, M \neq 2 N, 4 N, 6 N, \ldots$.

Proof. We may set $E=\kappa^{2}, z=\kappa r$ and $\psi(r)=\sqrt{z} \varphi(z)$ with

$$
\ell(\ell+1)=\gamma+\left(n+\frac{D-3}{2}\right)\left(n+\frac{D-1}{2}\right) .
$$

As long as equation (5) is solvable in terms of Bessel functions,

$$
\psi(r)=c_{1} \sqrt{r} H_{\nu}^{(1)}(\kappa r)+c_{2} \sqrt{r} H_{\nu}^{(2)}(\kappa r), \quad \nu=\ell+1 / 2
$$

we have to study their asymptotics in the asymptotic (i.e., $\varrho \gg 1$ ) wedges

- $\mathcal{S}_{0}=\left\{r=-\mathrm{i} \varrho e^{\mathrm{i} \varphi} \mid \varphi \in(-\pi / 2, \pi / 2)\right\}$,

- $\mathcal{S}_{ \pm k}=\left\{r=-\mathrm{i} e^{ \pm \mathrm{i} k \pi} \varrho e^{\mathrm{i} \varphi} \mid \varphi \in(-\pi / 2, \pi / 2)\right\}, k \geq 1$.

By construction, the tobogganic contour $\mathcal{C}^{(\alpha)}$ then connects the Stokes' wedge $\mathcal{S}_{0}$ with the Stokes' wedge $\mathcal{S}_{m}$ where $m=2 N$. Thus, we are entitled to use the well known asymptotic formulae

$$
\begin{aligned}
& \sqrt{\frac{\pi z}{2}} H_{\nu}^{(1)}(z) \exp \left[-\mathrm{i}\left(z-\frac{\pi(2 \nu+1)}{4}\right)\right]=1-\frac{\nu^{2}-1 / 4}{2 \mathrm{i} z}+\cdots, \\
& \sqrt{\frac{\pi z}{2}} H_{\nu}^{(2)}(z) \exp \left[\mathrm{i}\left(z-\frac{\pi(2 \nu+1)}{4}\right)\right]=1+\frac{\nu^{2}-1 / 4}{2 \mathrm{i} z}+\cdots,
\end{aligned}
$$

and conclude that in $\mathcal{S}_{2 k}$ we may eliminate the unphysical (i.e., asymptotically non-vanishing) component $H_{\nu}^{(1)}(z)$ and get the physical normalizable wave function proportional to $H_{\nu}^{(2)}(z)$. In $\mathcal{S}_{2 k+1}$, on the contrary, we encounter the physical $H_{\nu}^{(1)}(z)$ and unphysical $H_{\nu}^{(2)}(z)$. In this way we may start at $s=-\infty$ from

$$
\psi^{(\text {left })}(r)=c \sqrt{r} H_{\nu}^{(2)}(\kappa r) \quad\left(\text { with } r \in \mathcal{S}_{0}\right)
$$

and end up with $\psi^{(\text {right })}(r)$ given in closed form

$$
H_{\nu}^{(2)}\left(z e^{\mathrm{i} m \pi}\right)=\frac{\sin (1+m) \pi \nu}{\sin \pi \nu} H_{\nu}^{(2)}(z)+e^{\mathrm{i} \pi \nu} \frac{\sin m \pi \nu}{\sin \pi \nu} H_{\nu}^{(1)}(z) .
$$

Obviously, the latter function will vanish at $s=+\infty$ for the quantized angular momenta $\nu$ :

$$
m \nu=\text { integer }, \quad \nu \neq \text { integer } \Longrightarrow \quad \ell=\frac{M-N}{2 N} \text {. }
$$

From a purely pragmatic point of view the weakest point of the above, methodically important exactly solvable model of [31] lies in the complete absence of a confining external potential. An instability of this state with respect to random perturbations reflects just its being embedded in the continuum of scattering states. Unfortunately, the incorporation of any asymptotically confining force would already require the numerical solution of the corresponding tobogganic Schrödinger equation. 


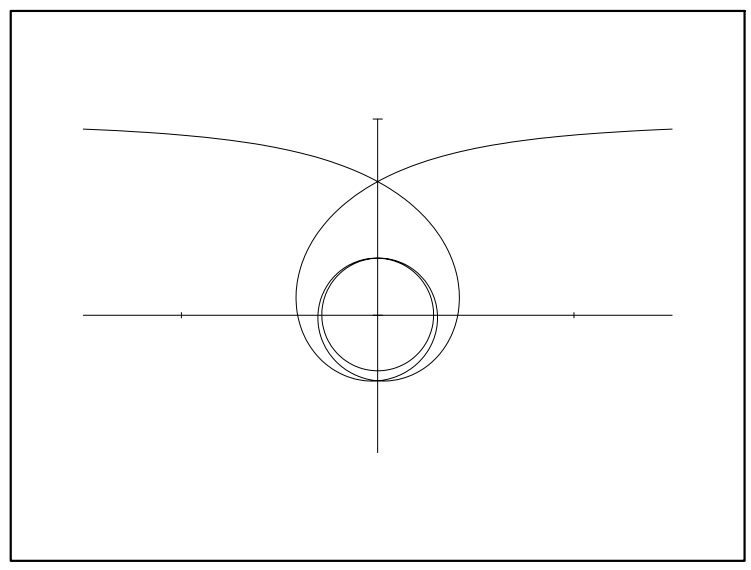

Figure 3. A tobogganic curve which three times encircles the branchpoint.

\section{The Sturm-Schrödinger "canonical" form of the toboggans}

Once we defined quantum toboggans as ordinary differential Schrödinger equations integrated over paths $\mathcal{C}(s)$ connecting several Riemann sheets of the wave functions $\psi(x)$, it is natural to complement, in the next step of the analysis, the single-loop tobogganic-curve of Fig. 2 by its multiply-spiralling descendants sampled, say, in Fig. 3. At the same time, in our present paper we shall not move to the next step of generalization in which more branch points are admitted in $\mathcal{R}$ (in this respect, e.g., [36] may be consulted).

For all of the left-right symmetric central spirals one can consider the tobogganic ordinary differential QTM Schrödinger equation

$$
H^{(1)} \psi_{n}^{(1)}(r)=E_{n} \psi_{n}^{(1)}(r), \quad H^{(1)}=-\frac{d^{2}}{d r^{2}}+V^{(1)}(r)
$$

and introduce a change of variables (say, $r \rightarrow y$ ) which is, up to some constants, such that $r \sim y^{\alpha}$ with a sufficiently large exponent $\alpha \in(1, \infty)$. Under such a mapping the two radial rays in the initial, tobogganic complex $r$-plane will be mapped on the two radial rays in the complex $y$-plane with a smaller angle between them. Thus, any angle between the radial rays becomes diminished by the mapping even when the initial rays are located on a multisheeted Riemann surface $\mathcal{R}$ of variable $r$. In this manner some of the rays which were originally hidden behind the cut (i.e., which belonged to the invisible Riemann sheets in variable $r$ ) will eventually move to the zeroth Riemann sheet in the new coordinate $y$, i.e., they become visible in the standard complex plane of $y$, say, with an upwards-running cut.

The detailed description of this technique which will rectify (i.e., straighten up) the QTM curves sampled in Figs. 2 and 3 (i.e., lower the total angle between their asymptotics) may be found elsewhere [32]. Here, let us only mention that in the literature one quite often finds the alternative, de-rectifying complex mappings $x \sim y^{\beta}$ with a small exponent $\beta \in(0,1)$. On Riemann surfaces this mapping acts in the opposite direction. Most often, it is used just to transform the exactly solvable harmonic oscillator into the exactly solvable bound-state part of the Coulombic (or Kepler) quantum system [37]. In the latter setting, both the initial and final coordinates remain real. The resulting, new Schrödinger equation for bound states acquires the form of the Sturmian eigenvalue problems where the energy appears multiplied by a non-constant (and, in our present paper, non-calligraphic-font) weight function $W(y)$,

$$
H^{(2)} \psi_{n}^{(2)}(y)=E_{n} W(y) \psi_{n}^{(2)}(y), \quad H^{(2)}=-\frac{d^{2}}{d y^{2}}+V^{(2)}(y) .
$$


In our present QTM setting we shall use $\alpha>1$ and emphasize that both the initial $r$ and final $y$ are complex in general. This means that in the respective usual and friendly or "false" Hilbert spaces $\mathcal{H}_{(1,2)}^{(F)}=\mathbb{L}_{2}(\mathcal{C})$ we have to deal with the manifestly non-self-adjoint operators,

$$
W \neq[W]^{\dagger} \quad \text { and } \quad H^{(1,2)} \neq\left[H^{(1,2)}\right]^{\dagger} \quad \text { in } \quad \mathcal{H}^{(F)}=\mathcal{H}_{(1,2)}^{(F)} .
$$

Just for illustration it is easy to verify that the change of variables

$$
\mathrm{i} x=(\mathrm{i} z)^{2}, \quad \psi_{n}(x)=\sqrt{z} \varphi_{n}(z)
$$

with $\alpha=2$ returns us from the first nontrivial tobogganic form of the standard harmonicoscillator Schrödinger equation to a non-tobogganic sextic-oscillator Sturm-Schrödinger equation $H \varphi=E W \varphi$ which remains manifestly $\mathcal{P} \mathcal{T}$-symmetric and defined on an U-shaped contour where $W>0$ [38],

$$
\left(-\frac{d^{2}}{d z^{2}}+4 z^{6}+\frac{4 \alpha^{2}-1 / 4}{z^{2}}\right) \varphi_{n}(z)=-4 E_{n} z^{2} \varphi_{n}(z)
$$

In general cases the rectification strategy is often further simplified and reduced just to the use of the winding-numbered paths of both the $r$ - or $y$-implementations

$$
\mathcal{C}^{(N)}(s)=-\mathrm{i}[\mathrm{i}(s-\mathrm{i} \varepsilon)]^{2 N+1}, \quad s \in(-\infty, \infty)
$$

with integer $N$. More details concerning the concrete realizations of this technique should be sought elsewhere (e.g., in our review paper [32]).

\section{Towards the non-Dirac metrics $\Theta \neq I$ in the physical Hilbert spaces of states}

\subsection{The non-Sturmian constructions with $W=I$}

The very brief account of the quantum theory using the non-standard (often called non-Dirac [13]) assumption (6) may be found in [15] or, in an even more compressed form, in Appendix A below. Its historical roots are in fact very recent since the explicit quantum-theoretical interpretation of $\mathcal{P} \mathcal{T}$-symmetric models as independently revealed by several groups of authors only dates back to the years 2001 and $2002[7,8,9]$.

It is necessary to add that the basic idea of the unphysical nature of the "friendly but false" Hilbert spaces $\mathcal{H}^{(F)}$ (with the trivial, often called Dirac's metric $\Theta^{(F)}=I$ ) and of the unitary equivalence between the alternative physical realizations $\mathcal{H}^{(S)}$ (with $\Theta^{(S)}=\Theta \neq I$ ) and $\mathcal{H}^{(P)}$ (with unfriendly Hamiltonians $\mathfrak{h}$ but still trivial $\Theta^{(P)}=I$ ) of the space of states already appeared in nuclear physics before 1992 [39].

Another marginal addendum to Appendix A concerns notation: one has to recollect that the physical Hilbert space $\mathcal{H}^{(S)}$ is defined as equipped with an amended Hermitian-conjugation operation (9) which co-applies also to the operators in the form

$$
\mathcal{A} \rightarrow \mathcal{A}^{\ddagger}:=\Theta^{-1} \mathcal{A}^{\dagger} \Theta
$$

such that we may deduce the reality of the spectrum of $H$ by its (crypto-)Hermiticity $H=H^{\ddagger}$ realized as "hidden" in space $\mathcal{H}^{(S)}$. Usually one proceeds in an opposite direction. Keeping in mind the definition of the conjugation $\mathcal{A}^{\ddagger}$ the main problem usually lies in the necessary construction of the metric operator $\Theta=\Theta^{\dagger}>I$.

As long as there exist many alternative operators $\Theta=\Theta(H)$ for a given Hamiltonian, different versions of the correct interpretation of the quantum system in question prove obtainable in general. In more detail, the situation is summarized in Appendix B below. 


\subsection{The transition to $W \neq I$}

There exist a few not too essential though still nontrivial differences between the non-tobogganic and tobogganic quantum systems. They may formally be reduced to the differences between the Schödinger and Sturm-Schrödinger time-independent equations where $W=I$ and $W \neq I$, respectively.

We now have to clarify the possibility and methods of construction of the metrics $\Theta$ for toboggans, say, in their rectified Sturm-Schrödinger representation. This representation might also be called "planarized" (i.e., defined in a single complex plane, admissibly with cuts), but we shall rather reserve the latter term for the narrower families of the models in which all the Riemann surface $\mathcal{R}$ is mapped on the single complex plane with a single cut or, alternatively, without any cuts at all.

Naturally, the latter specification of the concept of the "planarizability" would be much narrower, requiring that there is just a finite number of sheets forming the planarizable Riemann surface $\mathcal{R}$ in question. Even in such a form its use may still prove overcomplicated from the purely pragmatic point of view. For this reason, in what follows, we shall unify and further reduce the two alternatives and speak about the planarization-mapping correspondence $\mathcal{R} \leftrightarrow \mathbb{C}$ if and only if the mapping itself is given or known in an explicit form of some well-defined change of the variables.

The importance of distinguishing between the planarizable and non-planarizable QTM has not yet been addressed in the literature. This motivated also our present considerations in which we intend to emphasize that the concept of the planarizable Riemann surfaces is in fact not too robust (a small change of interaction may make them non-planarizable). At the same time, the corresponding expected enhanced sensitivity of the QTM spectra, say, to the small variations of the angular momenta $\ell$ is not too surprising. Indeed, the similar strong-sensitivity phenomenon has already been observed even in non-tobogganic models where it was quantitatively described, e.g., by Dorey et al. [35]. Unfortunately, its quantitative study in QTM context seems much more difficult. In this sense our present paper offers just a few first steps in this direction.

Our first message is that the key idea (attributed to Dyson [39]) of the use of the "non-unitary Fourier transformation" $\Omega$ and of the corresponding definition $\Theta=\Omega^{\dagger} \Omega$ of the metric proves transferrable to the QTM context in a rather straightforward manner.

In the more technical terms one has to start again from the doublet of Sturm-Schrödinger equations

$$
\left.\left.H|\lambda\rangle=\lambda W|\lambda\rangle, \quad H^{\dagger}\left|\lambda^{\prime}\right\rangle\right\rangle=\lambda^{\prime} W^{\dagger}\left|\lambda^{\prime}\right\rangle\right\rangle,
$$

where $\lambda$ denotes any element in the (by assumption, shared) spectrum of the "conjugate Hamiltonians" $H$ and $H^{\dagger}$.

Under another assumption of the reality and bound-state form ${ }^{1}$ of this spectrum the formulation of the general theory may again proceed in full analogy with the non-Sturmian and nontobogganic cases where $W=I$. In particular, besides the point spectrum (eigenvalues), due attention must also be paid to the possibility of the existence of the continuous spectrum as well as to the proofs of the absence of the residual part of the spectrum. Still, as long as our operators are mostly just the linear differential operators of second order, the rigorous discussion of these questions (plus of the questions of the domains, etc) remains as routine as in the non-tobogganic cases.

\subsection{Dyson-type mappings $\Omega \neq \Omega^{\dagger}$ for $W \neq I$}

Teaching by example let us return to the notation conventions of [15] and write

$$
|\psi \succ=\Omega| \psi\rangle, \quad|\psi\rangle \in \mathcal{V}, \quad \mid \psi \succ \in \mathcal{A}
$$

\footnotetext{
${ }^{1}$ I.e., discrete and bounded from below.
} 
for ket vectors in $\mathcal{H}^{(P)}$, taking into consideration also their duals,

$$
\prec \psi \mid=\langle\psi| \Omega^{\dagger} \in \mathcal{A}^{\prime}
$$

as well as the Hamiltonians $h=\Omega H \Omega^{-1}$ and the weight operators $w=\Omega W \Omega^{-1}$ defined as acting in $\mathcal{H}^{(P)}$.

In this language the doublet of the Dieudonné-type generalized constraints

$$
h^{\dagger}=\left(\Omega^{-1}\right)^{\dagger} H^{\dagger} \Omega^{\dagger}=h,
$$

and

$$
w^{\dagger}=\left(\Omega^{-1}\right)^{\dagger} W^{\dagger} \Omega^{\dagger}=w,
$$

implies that we get, after a trivial re-arrangement, the pair of equations

$$
H^{\dagger}=\Theta H \Theta^{-1}, \quad W^{\dagger}=\Theta W \Theta^{-1}, \quad \Theta=\Omega^{\dagger} \Omega .
$$

They are defined directly in the unphysical and auxiliary but, presumably, maximally computation-friendly space $\mathcal{H}^{(F)}$.

The further development of the theory is more or less obvious - a few further technical details are made available in Appendix B below.

\section{The planarized toboggans}

In the light of the results described in Appendix $\mathrm{C}$ the physical source of the reliability of the large- $\ell$ spectral estimates of [40] might be seen in the large magnitude of the distance (i.e., of the length of the path on which the differential expression is defined) between the branch-point singularity and the position of the minimum/minima of the related effective potential.

We now intend to emphasize that the interaction-dependent multiplicity of the latter minima [41] together with the comparability of their respective distances from the branch point indicates that one must be very careful with any a priori extrapolation of any non-QTM observation to its possible QTM analogues.

The latter warning led us also to our present proposal of restricting one's attention solely to the models in which one could eliminate the multisheeted form of $\mathcal{R}$ by the purely analytic means of the the above-discussed rectification transformation.

As long as we are dealing here just with the models possessing a mere single branch-point singularity we may work with the necessary rectification changes of variables in closed form. As a consequence, we intend to achieve a maximal profit from the maximalized simplicity of the replacement of the tobogganic version $H|\psi\rangle=E|\psi\rangle$ of Schrödinger equation by its single-plane Sturm-Schrödinger eigenvalue counterpart $H|\psi\rangle=E W|\psi\rangle$ containing its energy in the term with a weight factor $W \neq I$. In other words, we shall restrict our choice of dynamics by the formal requirement that the latter equation does not contain any strong singularities anymore.

On this background we shall return to the idea of the possible QTM implementations of the supersymmetric partnership which has been originally discouraged by our recent general study [19] where we did not assume any planarizability of the QTM Riemann sheet $\mathcal{R}$.

\subsection{Asymptotically harmonic oscillator as the simplest planarizable toboggan}

Let us assume that our potential is asymptotically quadratic, i.e., $V(x)=x^{2}+\mathcal{O}(x)$ at the large complex $x$. Then the most general form of the wave function $\psi(x) \sim c_{+} e^{x^{2} / 2}+c_{-} e^{-x^{2} / 2}$ asymptotically vanishes (and "remains physical") either at $c_{+}=0$ (this takes place either in the 


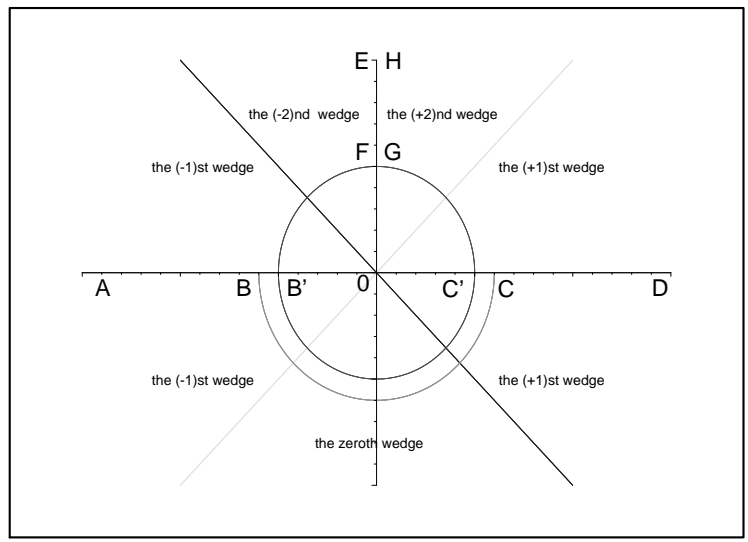

Figure 4. The typical harmonic-oscillator integration curves visible on the first Riemann sheet of complex $x$ (see the text for a detailed explanation). The upwards-running cut starts at the origin; arbitrary units.

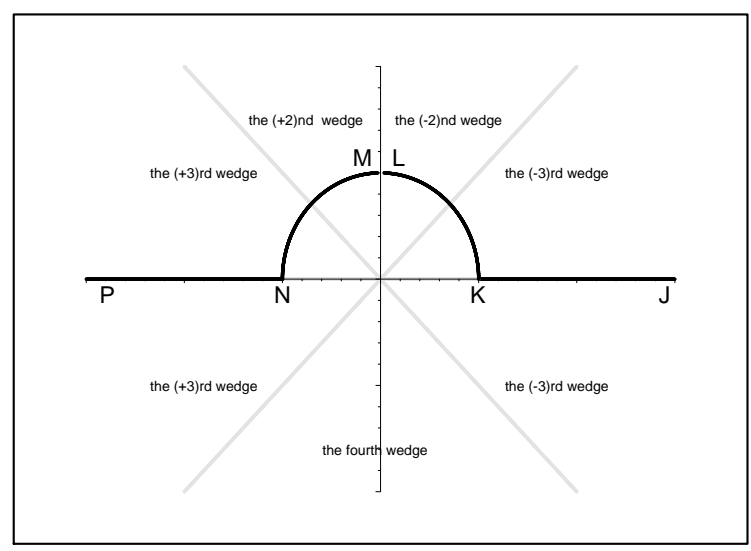

Figure 5. The beginning (J-K-L) and the end (M-N-P) of the unrectified tobogganic curve lying on the second Riemann sheet. The upwards-running cut starts at the origin; arbitrary units.

$( \pm 1)$ st Stokes wedges of Fig. 4 or in the $( \pm 3)$ rd Stokes wedges of Fig. 5$)$ or at $c_{-}=0$ (this takes place either in the zeroth and $( \pm 2)$ nd Stokes wedges of Figs. 4 and 5 or in the last, $(+4)$ th $=$ $(-4)$ th Stokes wedge of Fig. 5).

Let us assume, in addition, that the Riemann surface $\mathcal{R}$ of analyticity of our eligible physical wave functions $\psi^{(+)}(x)=e^{+x^{2} / 2+\mathcal{O}(x)}$ or $\psi^{(-)}(x)=e^{-x^{2} / 2+\mathcal{O}(x)}$ is just two-sheeted, i.e., that near the origin we have $\psi(x) \sim x^{J / 2}$ with an odd integer $J$.

For the sake of definiteness, we may pick up angular momentum $\ell=1 / 2$ giving $J=3$. Then, the elementary change of variables $i x=(i y)^{2}$ (with full details either easily derived or taken, say, from [32]) gives the new, equivalent form of wave function $\varphi(y)$ which is, by our assumption, regular or at most weakly singular in the origin. This means that the change of variables $x \rightarrow y$ maps the two-sheeted Riemann surface ${ }^{2} \mathcal{R}$ onto the single "target" complex plane $\mathbb{C}$ of Fig. 6 which, formally, plays the role of a single-sheeted Riemann surface of the new, "Sturmianic" [42] wave function $\varphi(y)$.

By construction of our particular illustrative example, there are no singularities and no cuts in the latter complex plane. For the sake of simplicity the boundaries of the Stokes wedges were also omitted in Fig. 6 since they only prove relevant in the asymptotic domain where $|y| \rightarrow \infty$.

\footnotetext{
${ }^{2}$ Shown in Figs. 4 and 5 and pertaining to our "initial", tobogganic as well as non-tobogganic, asymptotically harmonic-oscillator-like wave functions $\psi(x)$.
} 


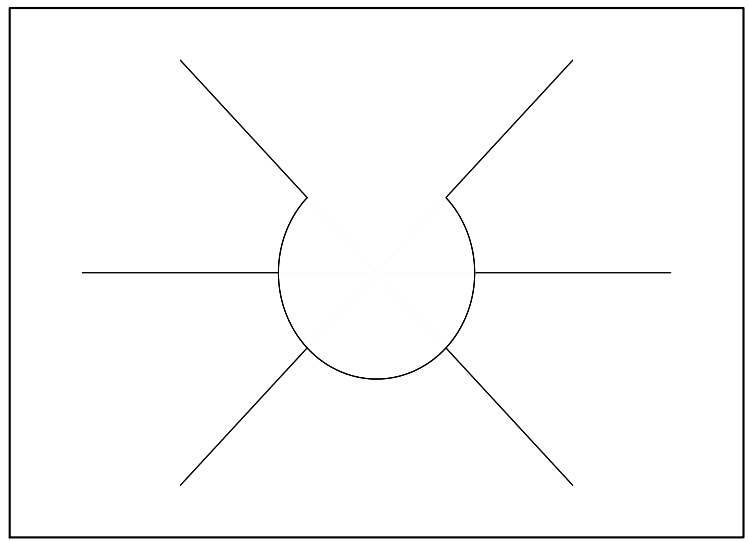

Figure 6. Complex $y$-plane and the planarized triplet of $x$-paths of Figs. 4 and 5.

\subsection{The planarizable supersymmetric toboggans}

Once we return, in a climax of our preceding QTM-related exposition and considerations, to the problem of the possible SUSY partnership between strongly singular potentials, we may now feel confident that the formalism is prepared for the rigorous regularization treatment of the changes of the strength of the singularities in the potentials.

For the sake of definiteness let us start from equation (1) and assume that the generic analytic wave functions $\psi(r)$ of the quantum system in question exhibit a general power-law behavior in the origin,

$$
\psi_{0}^{(\gamma)}(r) \approx r^{\gamma+1 / 2}, \quad|r| \ll 1, \quad \gamma \in \mathbb{R} .
$$

This implies that the related Riemann surface $\mathcal{R}$ will be multisheeted in general, and $K$-sheeted (with $K=K(\gamma)<\infty$ ) for special $\gamma=$ rational. This is one of the facts which make the problem of QTM spectra rather nontrivial in general, and this is also one of the reasons why we decided to pay here attention to the models with finite $K(\gamma)$. These models find a natural rectificationtransformation regularization which eliminates the branch point(s) and "planarizes" the model in the way explained above.

In any case, our "calligraphic-font" superpotential of equation (1) acquires now one of the most elementary singular forms,

$$
\mathcal{W}(r)=\mathcal{W}^{(\gamma)}(r)=-\frac{\partial_{r} \psi_{0}^{(\gamma)}(r)}{\psi_{0}^{(\gamma)}(r)}=\chi(r)-\frac{\gamma+1 / 2}{r},
$$

where $\chi(r)$ may be an arbitrary function which is sufficiently regular in the origin. Thus, we know the doublet of operators $A=\partial_{q}+\mathcal{W}$ and $B=-\partial_{q}+\mathcal{W}$ and easily deduce the explicit form of the "upper" and "lower" sub-Hamiltonians in equation (2),

$$
H^{(U)}=B \cdot A=\hat{p}^{2}+\mathcal{W}^{2}-\mathcal{W}^{\prime}, \quad H^{(L)}=A \cdot B=\hat{p}^{2}+\mathcal{W}^{2}+\mathcal{W}^{\prime} .
$$

Near the origin, their dominant parts read as follows,

$$
H^{(U)}=\hat{p}^{2}+\frac{\gamma^{2}-1 / 4}{r^{2}}+\mathcal{O}\left(\frac{1}{r}\right), \quad H^{(L)}=\hat{p}^{2}+\frac{(\gamma+1)^{2}-1 / 4}{r^{2}}+\mathcal{O}\left(\frac{1}{r}\right)
$$

We see that the SUSY correspondence between operators $H^{(U)}$ and $H^{(L)}$ leaves our auxiliary integer quantity $K(\gamma)$ invariant. This implies that in any QTM version (i.e., independently of 


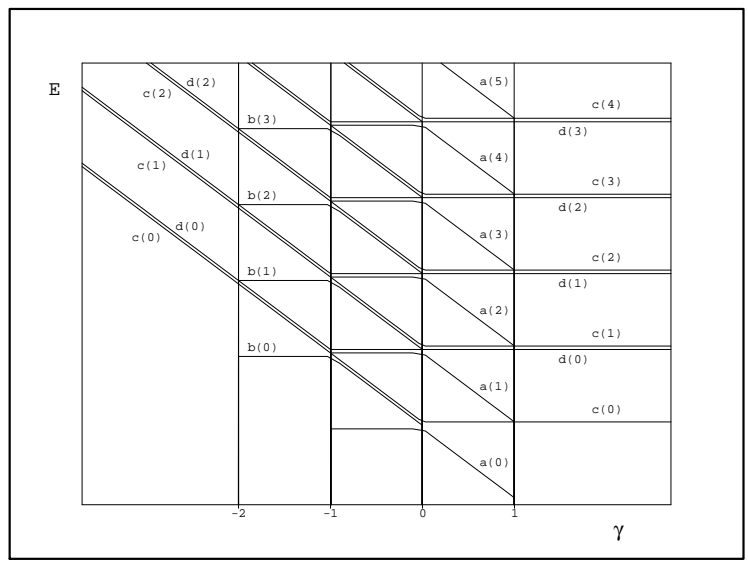

Figure 7. The $\gamma$-dependence of the spectrum of the supersymmetric model which is generated by the singular superpotential (7) where $\chi(r)=r$.

the winding number $N$ ), both of the SUSY-partner QTM operators may be made regular and planar (=planarized) simultaneously.

An important remark should be added here since for the real $\gamma$ we have $H^{(U)}$ and $H^{(L)}$ which depend on the mere absolute values of $|\gamma|$ and $|\gamma+1|$, respectively. This implies the emergence of various interesting structures and asymmetries in the spectra. Some of them were described, in [24], via the exactly solvable model with linear $\chi(r)=r$. Some of the other features of this harmonic-oscillator-like solvable toy model were discussed in proceedings [43]. Unfortunately, due to an error in printing, the illustrative spectrum did not appear in the paper. Fortunately, this information has not lost its appeal with time so we now present it via Fig. 7 at last. What we see in this picture are

1) the "far left" energies which are completely degenerate,

$$
E_{N}^{(U)}[\equiv c(N)]=E_{N}^{(L)}[\equiv d(N)], \quad \gamma \in(-\infty,-2)
$$

2) the "near left" energy levels with $\gamma \in(-2,-1)$, exhibiting a Jevicki-Rodrigues-like breakdown of SUSY [2] since the new levels $E_{N}^{(L)}[\equiv b(N)]$ remain non-degenerate;

3) the "central-domain" levels with $\gamma \in(-1,0)$,

$$
E_{N}^{(U)}[\equiv a(N)]<E_{N}^{(L)}[\equiv b(N)]=E_{N}^{(U)}[\equiv c(N)]<E_{N}^{(L)}[\equiv d(N)]=a(N+1)<\cdots .
$$

Note that one obtains the usual harmonic oscillator at $\gamma=-1 / 2$, plus its smooth extension which ceased to be equidistant - still, the SUSY is unbroken;

4) the "near right" domain of $\gamma \in(0,1)$ where the series $E_{N}^{(L)}[\equiv b(N)]$ ceases to exist;

5) the "far right" spectrum with $\gamma \in(1, \infty)$ and degeneracy

$$
E_{(L)}^{(+\alpha)}[\equiv c(N)]=d(N-1), \quad N>0 .
$$

Another concluding remark should be made on the complex-conjugation-mimicking operators $\mathcal{T}$ as introduced in [19]. Their role has been recalled here via equations (3) and (4). Obviously, in a planarized representation of any QTM and/or SUSY QTM system, these operators will mediate certain complex rotations by angles $\varphi=2 \pi / K(\gamma)$. This means that they will operate as mutual mappings between the neighboring rectified paths of the complex coordinates $y=\hat{\mathcal{C}}(s)$ in the single-sheeted $y$-plane without any cuts. 


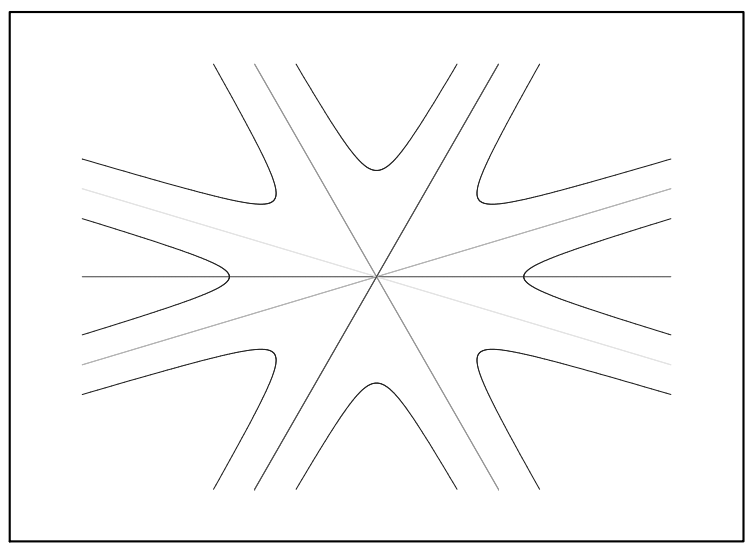

Figure 8. A sample of the planarized system of the eight QTM trajectories of $y \in \mathcal{T}^{n} \hat{\mathcal{C}}(s)$.

The sequence of the individual maps $\mathcal{T} \hat{\mathcal{C}}(s), \mathcal{T}^{2} \hat{\mathcal{C}}(s), \mathcal{T}^{3} \hat{\mathcal{C}}(s), \ldots$ may be represented, schematically, by the hyperbolas in Fig. 8 where we assumed that the whole QTM system has been planarized (i.e., there is no cut). Obviously, this picture strongly suggests that the quantitative analysis of the spectra will probably exhibit a growing difficulty with the growth of the index $K(\gamma)=0,1, \ldots$ which characterizes a "hidden" topology and symmetry of the QTM system.

\section{Conclusions}

In the mathematical language of operator theory on can say that the QTM (or, if you wish, also $\mathcal{P} \mathcal{T}$-symmetric and other) Hamiltonians are similar to Hermitian operators [44]. In the literature one might find a lot of rigorous results in this direction (pars pro toto let us mention the brief note by Langer and Tretter [45] from 2004).

In the equally rigorous language of integrable models, the knowledge and an appropriate reinterpretation of the IM/ODE correspondence did even provide one of the most valuable proofs of reality of the spectra from $H \neq H^{\dagger}$ (cf. [10]).

Incidentally, the similar though much easier (and, hence, less often cited) proofs of the reality of spectra also emerged in the domain of the (new) exactly solvable models - systematic study [46] may be read as a collection of examples with further references.

On a purely heuristic level one of the most efficient formal features of the candidates $H \neq H^{\dagger}$ for Hamiltonians must be seen in their $\mathcal{P} \mathcal{T}$-symmetry, i.e., in the peculiar intertwining property $H^{\dagger} \mathcal{P}=\mathcal{P} H$ where $\mathcal{P}$ is, unexpectedly often, an operator of (spatial) parity.

One should not forget that the boom of interest in $\mathcal{P} \mathcal{T}$-symmetric models grew even beyond the boundaries of quantum theory, viz, to various non-quantum applications of toy models $H \neq H^{\dagger}$ in magnetohydrodynamics (cf. Günther et al. [47]) or in optics (Rüter et al. [48]) etc.

In a parallel recent history of development of the purely pragmatic use of the concepts of supersymmetry in Quantum Mechanics (cf., e.g., [24]) one may notice the existence of the regular turns of emphasis from the appeal of the underlying algebra of operators to the necessity of studying the spaces of wave functions and the related boundary conditions. In this sense we consider the possibility of construction of SUSY QM models as systems living on topologically nontrivial Riemann surfaces [19] most appealing.

In our present considerations we used this formalism for addressing, directly, the family of problems related to the names of Jevicki and Rodrigues [2]. As long as these problems emerge, as a rule, in connection with the emergence of singular interaction potentials, their potential relevance in physics is undeniable. Nevertheless, our present text also emphasized the presence of many technical obstacles in this effort. 
In summary, the present innovative use of the complexified coordinates $x$ (or $y$ ) and of the non-standard, non-Hermitian operators in SUSY context may be expected to throw new light on the multiple connections between the abstract algebras (sampled by SUSY) and nonlinear symmetries (sampled here by $\mathcal{P} \mathcal{T}$-symmetry), especially in their concrete transparent representations in terms of the ordinary differential operators.

Needless to add that our text still left multiple questions entirely open. Still, some of these questions (ranging from the abstract theories down to very concrete estimates and calculations) cannot remain unanswered in a long perspective, provided that we wish to employ the proposed concepts in a more concrete phenomenological context. For this purpose we expect that the future research will move towards a deeper synthesis of the algebraic study of the foundations of SUSY QM with the truly analytic-function approaches to the mathematical as well as physical puzzles connected with the models living on more than one sheet of a Riemann surface of the (presumably, analytic) wave functions.

\section{A Hilbert spaces endowed with nontrivial inner products}

Among applications of quantum theory one only rarely encounters unusual models like, e.g., the quantum clock in which the quantum observable is the time [49]. The wealth of possibilities offered by the abstract formalism is disproportionately often reduced just to the description of the one-dimensional motion of a point (quasi)particle. The measurement is then understood to be the measurement of position $x$ while the evolution of the wave function $\psi(x)$ in time is assumed controlled (i.e., generated) by the Hamiltonian $H=-d^{2} / d x^{2}+V(x)$ where $H_{0}=-d^{2} / d x^{2}$ represents kinetic energy and where a real function $V(x)$ mimics the effects of an external medium.

In such a context Bender and Boettcher [6] evoked a lot of discussions by considering a oneparametric set of purely imaginary functions $V^{(\delta)}(x)=x^{2}(\mathrm{i} x)^{\delta}$ with arbitrary real $\delta \geq 0$ and by disentangling, simultaneously, any connection between the measurable values of position $q \in \mathbb{R}$ and the admissible values of the variable $x \in \mathcal{C}(s)$ in $\psi(x)$. Here, the real parameter $s \in \mathbb{R}$ just parametrized an ad hoc complex curve such that $\mathcal{C}(s) \neq \mathbb{R}$ in general (cf., e.g., review paper [13] for more details).

In such a setting the concept of quantum toboggans has been introduced by the mere replacement of condition $\mathcal{C}(s) \in \mathbb{C}$ by condition $\mathcal{C}(s) \in \mathcal{R}$ where symbol $\mathcal{R}$ represents a nontrivial, multisheeted Riemann surface pertaining to wave functions $\psi(x)$. In this case the curves $\mathcal{C}(s)$ may be spiral-shaped (hence the name), and only their topology must be compatible with the domains of analyticity of the underlying time-independent Schrödinger equation

$$
H \psi(x)=E \psi(x), \quad H=-\frac{d^{2}}{d x^{2}}+V(x) b, \quad x \in \mathcal{C}(s) \subset \mathbb{C}, \quad s \in \mathbb{R} .
$$

For any practical (e.g., phenomenological) purposes one then has the full freedom of making use of the analyticity properties of $V(x)$ and $\psi(x)$. Strictly in the spirit of [6] (or even of the older futuristic remark [50]) one may really convert the same differential equation (8) into many non-equivalent spectral problems by the mere variation of the underlying (classes of) curves $\mathcal{C}(s) \in \mathcal{R}$.

From such an observation one has to extract the following two messages. First, one has to accept the idea that the information about dynamics may be carried not only by the differential expressions $H$ but also by the topological properties of the curves $\mathcal{C}(s)$. Second, once we lost the identification of $x$ with the position, we have to reopen the problem of the concrete realization of the preparation of the system at some initial time $t=0$ as well as of the specification of the correct physical content of the measurement over the system at some subsequent, final time $t=T>0$. 
Both these questions were already addressed, in the context of nuclear physics, by Scholtz et al. [39]. In the similar, albeit more realistic physical setting these authors immediately realized that whenever one has, in the usual mathematical sense, $H \neq H^{\dagger}$, one has to leave the corresponding "usual" Hilbert-space representation $\mathbb{L}^{2}$ of the states (based on the use of the "usual" inner product $\left.\langle\phi \mid \psi\rangle=\int_{\mathbb{R}} \phi^{*}(x) \psi(x) d x\right)$ by a non-equivalent, adapted, "standard" Hilbert-space representation denoted, say, by the symbol $\mathcal{H}^{(S)}$ according to our recent brief review [15].

In the spirit of the abstract quantum theory the use of the latter space $\mathcal{H}^{(S)}$ returns us to the textbook quantum theory. In particular, this space must render our given Hamiltonian self-adjoint, i.e., it must be endowed by an amended, "metric-dependent" inner product, $\langle\phi \mid \psi\rangle \rightarrow\langle\phi|\Theta| \psi\rangle$. The Hilbert-space-metric operator $\Theta$ itself must satisfy a certain set of natural mathematical requirements of course [39].

Starting from the very early applications of the latter idea people often tried to avoid the direct use of the correct physical space $\mathcal{H}^{(S)}$ endowed with the nontrivial metric $\Theta$. Indeed the work within this space requires not only a suitable modification of the related Dirac-notation conventions (in this respect we shall follow here the recommendations given in [15]) but also a mind-boggling necessity of using the following "corrected" definition of the Hermitian conjugation:

$$
\mathcal{T}:|\psi\rangle \rightarrow\langle\langle\psi|:=\langle\psi| \Theta .
$$

A comparatively easy insight in this rule is obtained when one factorizes $\Theta=\Omega^{\dagger} \Omega$ and replaces the physical space $\mathcal{H}^{(S)}$ of kets $|\psi\rangle$ and (doubled) bras $\langle\langle\psi|$ by its unitarily equivalent "partner" Hilbert space $\mathcal{H}^{(P)}$ of kets

$$
\left.\left|\psi^{(P)}\right\rangle:=|\psi \succ:=\Omega| \psi\right\rangle
$$

and of bras

$$
\left\langle\psi^{(P)}|:=\prec \psi|:=\left\langle\langle\psi| \Omega^{-1} .\right.\right.
$$

On a purely abstract level of thinking one might appreciate that inside $\mathcal{H}^{(P)}$ the metric is trivial again, $\Theta^{(P)}=I$. At the same time the price for simplification is too high in general since the Hamiltonian itself acquires a prohibitively complicated transformed form $\mathfrak{h}=\Omega H \Omega^{-1}$.

We may summarize that in the majority of applications of models with apparent nonHermiticity rule $H \neq H^{\dagger}$ valid inside the "friendly" but "false" space $\mathbb{L}^{2} \equiv \mathcal{H}^{(F)}$, the concrete form of the above mentioned "non-unitary Fourier" [5] or "Dyson's" [39] mapping $\Omega \neq\left(\Omega^{\dagger}\right)^{-1}$ is both ambiguous and virtually impossible to construct. A persuasive confirmation of this generic rule may be found in [51] where, up to the third order perturbation corrections, all of the eligible operators $\Omega$ were constructed for the "small" imaginary cubic potential $V(x)=\lambda \mathrm{i} x^{3}$.

It is worth adding that the ambiguity of the "admissible" metric operators $\Theta=\Theta(H)$ represents in fact a very unpleasant feature of the theory. For this reason, its entirely general factorization $\Theta=\Omega^{\dagger} \Omega$ in terms of the so called Dyson's mappings $\Omega$ [39] is quite often being reduced and replaced by its more specific and less general forms ${ }^{3}$.

\section{B The three-Hilbert-space formulation of quantum mechanics for quantum toboggans}

On the overall background of the three-Hilbert-space formulation of quantum mechanics as given in [15] we should emphasize, at the very beginning, that there exist many important technical

\footnotetext{
${ }^{3}$ To name just a few let us recollect the parity-using factorizations $\Theta=\mathcal{C P}$ (= the most popular ansatz using a "charge" $\mathcal{C}$ such that $\mathcal{C}^{2}=I$, cf. [9]) and $\Theta=\mathcal{P} \mathcal{Q}$ (where $Q$ denotes quasi-parity, cf. [7]) - or simply the square-root factorization $\Theta=\varrho^{2}$ with a special, self-adjoint $\varrho=\varrho^{\dagger}-$ cf. [14] or [52].
} 
details of its presentation which will vary with the assumptions concerning the spectral properties of the operators in question. For this reason let us reduce the corresponding rigorous discussion to the necessary minimum and assume that all of the operators of our present interest possess just the point spectrum (composed of a finite or countably infinite number of eigenvalues and bounded from below) and no continuous or residual spectrum.

This assumption will certainly help us to simplify also the language and to use just the "minimally" modified version of the standard Dirac's notation as described in more detail in [15]. In particular, let us recollect that the triple possibility of representation of the ket vectors may be summarized in the following, manifestly time-dependent picture inspired by the very first proposal and application [39] of the whole, and very original, scheme in nuclear physics,

\begin{tabular}{|c|c|c|c|}
\hline $\begin{array}{r}\text { phys } \\
\text { difficul }\end{array}$ & $\begin{array}{l}\text { al } \mathcal{H}^{(\mathrm{P})}(\text { fer } \\
\mid \psi(t) \succ \equiv \Omega(\end{array}$ & $\begin{array}{l}\text { ions }) \\
|\psi(t)\rangle\end{array}$ & \\
\hline Dyson's map $\Omega(t)$ & & & $\operatorname{map} \Omega^{-1}(t)$ \\
\hline $\begin{array}{c}\text { friendlier } \mathcal{H}^{(\mathrm{F})} \text { (bosons) } \\
|\psi(t)\rangle=\text { computable }\end{array}$ & $\operatorname{map} \stackrel{\Omega \Omega^{-1}}{\longrightarrow}=I$ & $\begin{array}{l}\text { stande } \\
\qquad \mid \psi\end{array}$ & $\begin{array}{l}\text { ard } \mathcal{H}^{(\mathrm{S})} \text { (bosons) } \\
(t)\rangle \text { the same }\end{array}$ \\
\hline
\end{tabular}

In this context the key innovation was twofold. Firstly, the Fourier-like mapping $\Omega$ (proposed, presumably, by Dyson) was allowed to be non-unitary. Secondly, the following arramengement of the triplet of related, Hilbert-space-dependent bra vectors proved rather unusual, showing

\begin{tabular}{|c|c|c|}
\hline & $\begin{array}{l}\text { physical } \prec \psi(t) \mid \in\left(\mathcal{H}^{(\mathrm{P})}\right)^{\prime} \\
=\text { prohibitively complicated }\end{array}$ & $\begin{array}{l}(\mathrm{P}))^{\prime} \\
\text { cated }\end{array}$ \\
\hline $\operatorname{map} \Omega^{\dagger}(t)$ & & $\searrow \quad \operatorname{map} \Omega(t)$ \\
\hline $\begin{aligned} & \langle\psi(t)| \in\left(\mathcal{H}^{(\mathrm{F})}\right)^{\prime} \\
= & \text { inconsistent physics }\end{aligned}$ & map $\stackrel{\Theta(t)=\Omega^{\dagger} \Omega \neq I}{\longrightarrow}$ & $\begin{aligned} & \left\langle\langle\psi(t)| \in\left(\mathcal{H}^{(\mathrm{S})}\right)^{\prime}\right. \\
= & \text { innovated conjugation }\end{aligned}$ \\
\hline
\end{tabular}

Two conclusions may be deduced. Firstly, the mapping $\mathcal{T}:\langle\psi| \rightarrow\langle\langle\psi|=\langle\psi| \Theta$ may be perceived as a "very natural and very physical" or "amended" Hermitian conjugation (reason: the Hamiltonian s made self-adjoint in $\left.\mathcal{H}^{(\mathrm{S})}\right)$. Secondly, the "double-bra" vectors may be constructed as eigenvectors of the no-metric-conjugate $H^{\dagger}$ (which differs from $H$ itself). Then, one can reconstruct the metric using the explicit formula

$$
\left.\Theta=\sum|\psi\rangle\right\rangle\langle\langle\psi|
$$

\section{B.1 The hermitized "generalized eigenvalue problems" $(W \neq I)$}

Inside the constructively inaccessible "paternal", physical Hilbert space $\mathcal{H}^{(\mathrm{P})}$ our Sturm-Schrödinger equation is self-adjont,

$$
h|\lambda \succ=\lambda w| \lambda \succ .
$$

Inside the same space we may work with the Sturmianic orthogonality relations

$$
\prec \lambda|w| \lambda^{\prime} \succ=\prec \lambda|w| \lambda \succ \cdot \delta_{\lambda, \lambda^{\prime}}
$$

and with the Sturmianic completeness relations,

$$
I=\sum_{\lambda}\left|\lambda \succ \frac{1}{\prec \lambda|w| \lambda \succ} \prec \lambda\right| w .
$$


In the same manner there exists the following Sturmianic spectral representation of the Hamiltonian,

$$
h=\sum_{\lambda} w\left|\lambda \succ \frac{\lambda}{\prec \lambda|w| \lambda \succ} \prec \lambda\right| w .
$$

\section{B.2 Back to $\mathcal{H}^{(F)}$}

As long as the manifestly unphysical and "false" Hilbert space $\mathcal{H}^{(\mathrm{F})}$ is still, by assumption, maximally "friendly" to computations, it makes sense to translate the above-listed formulae to this space as well. Thus, one arrives at the orthogonality relations in $\mathcal{H}^{(F)}$,

$$
\left\langle\lambda\left|\Omega^{\dagger} w \Omega\right| \lambda^{\prime}\right\rangle=\left\langle\lambda|\Theta W| \lambda^{\prime}\right\rangle=\langle\lambda|\Theta W| \lambda\rangle \cdot \delta_{\lambda, \lambda^{\prime}}
$$

as well as at the following completeness relations inside $\mathcal{H}^{(F)}$,

$$
I=\sum_{\lambda}|\lambda\rangle \frac{1}{\langle\lambda|\Theta W| \lambda\rangle}\langle\lambda| \Theta W
$$

Thirdly, the spectral decomposition of the Hamiltonian in $\mathcal{H}^{(F)}$ has the following form,

$$
H=\sum_{\lambda} W|\lambda\rangle \frac{\lambda}{\langle\lambda|\Theta W| \lambda\rangle}\langle\lambda| \Theta W
$$

Although the choice of the convention $|\lambda\rangle\rangle=\Theta|\lambda\rangle$ represents just one of many alternative possibilities, its preference also simplifies the normalization of the overlap $\langle\lambda|\Theta W| \lambda\rangle=1$.

\section{B.3 $\Theta W$ stays positive definite}

It is necessary to keep in mind that the use of the formal abbreviations

$$
\left.\mid \psi\}=W|\psi\rangle, \quad \mid \psi\}\}=W^{\dagger}|\psi\rangle\right\rangle
$$

may perceivably simplify our formulae. First, it leads to an amended orthogonality,

$$
\left\langle\lambda|\Theta W| \lambda^{\prime}\right\rangle=\left\{\left\{\lambda\left|\lambda^{\prime}\right\rangle=\left\langle\langle\lambda| \lambda^{\prime}\right\}=\delta_{\lambda, \lambda^{\prime}} .\right.\right.
$$

Second, the alternative completeness relations read

$$
I=\sum_{\lambda}|\lambda\rangle\left\{\left\{\lambda\left|=\sum_{\lambda}\right| \lambda\right\}\langle\langle\lambda| .\right.
$$

Third, the spectral-representation expansions

$$
\left.W=\sum_{\lambda} \mid \lambda\right\}\left\{\left\{\lambda\left|, \quad H=\sum_{\lambda}\right| \lambda\right\} \lambda\{\{\lambda \mid\right.
$$

represent, this time, not only the Hamiltonians but also the weight-operators.

In a final step of our review of the formalism we may rewrite the Dieudonné equations in the form

$$
\left.\left.\sum_{\lambda} \mid \lambda\right\}\right\} \lambda\left\{\lambda\left|\Theta=\sum_{\lambda} \Theta\right| \lambda\right\} \lambda\{\{\lambda \mid
$$

or

$$
\left.\left.\sum_{\lambda} \mid \lambda\right\}\right\}\left\{\lambda\left|\Theta=\sum_{\lambda} \Theta\right| \lambda\right\}\{\{\lambda \mid
$$

and easily derive the final single-series spectral formula

$$
\left.\Theta=\sum_{\lambda}|\lambda\rangle\right\rangle\{\{\lambda \mid
$$

for the metric [42]. 


\section{Imaginary cubic toboggans in the large- $L$ perturbation regime}

For readers interested in the practical and phenomenological aspects of our present considerations let us briefly recall the results of calculations [40] where the popular [51] non-Hermitian potential $V(x)=i x^{3}$ has been studied in the QTM kinematical regime and in the high-angularmomentum dynamical domain.

First, the rectification change of variables did lead to the Schrödinger (or rather SturmSchrödinger) bound-state problem

$$
\left[-\frac{d^{2}}{d y^{2}}+\frac{L(L+1)}{y^{2}}+\mathrm{i}(-1)^{N}(2 N+1)^{2} y^{10 N+3}\right] \psi_{n}(y)=(2 N+1)^{2} y^{4 N} E_{n}^{[N]} \psi_{n}(y),
$$

where $N$ is a small integer while $L$ is only assumed real and (very) large. We are returning to these results because they very well illustrate not only the method (of asymptotic estimates) but also the comparatively friendly nature of the spectral consequences of the topological nontriviality of the QTM integration paths $\mathcal{C}=\mathcal{C}^{(N)}$ where, roughly speaking, the small and positive integer $N$ is a winding number of the tobogganic path.

\section{C.1 The method: $1 / L$ series at $N=0\left(L=\ell, y=q^{(0)}\right)$}

Near a (local as well as global) minimum $V_{\text {eff }}(Q)$ of any sufficiently smooth function $V_{\text {eff }}(q)$ we may often work just with the truncated forms of the Taylor series

$$
V_{\text {eff }}(q)=V_{\text {eff }}(Q)+\frac{1}{2} V_{\text {eff }}^{\prime \prime}(q) \xi^{2}+\frac{1}{6} V_{\text {eff }}^{\prime \prime \prime}(q) \xi^{3}+\cdots,
$$

where $\xi=q-Q$ and where we showed in [40] that for the imaginary cubic oscillators one may select $2 \ell(\ell+1)=3 \mathrm{i} Q^{5}=$ large and restrict the set of all of the available (complex) roots $Q=Q_{j}$, $j=1,2, \ldots, 5$ just to the $j=1$ preferred item. Then we deduced

Lemma 1. In the non-tobogganic case the low-lying imaginary-cubic spectrum is prescribed by the estimate

$$
E_{n}^{[0]}=-\frac{5 \tau^{3}}{2}+\sqrt{\frac{15 \tau}{2}}(2 n+1)+\mathcal{O}\left(\tau^{-3 / 4}\right), \quad n=1,2, \ldots
$$

where

$$
\tau=\left|\left(\frac{2}{3} \ell(\ell+1)\right)^{1 / 5}\right| \quad \text { in } \quad Q_{j}=-\mathrm{i} \tau \exp \left(\frac{2 \mathrm{i} \pi(j-1)}{5}\right), \quad j=1,2, \ldots, 5 .
$$

Proof. With $j=1$ and with a small $\sigma=1 / \tau^{1 / 4}$ we may rescale $\xi \rightarrow \sigma \xi$ and re-write

$$
V_{\text {eff }}(q)=-\frac{5}{2} \tau^{3}+\frac{15 \tau}{2} \xi^{2}-5 \mathrm{i} \xi^{3}+\mathcal{O}\left(\tau^{-1}\right)
$$

leading to the leading-order harmonic-oscillator Schrödinger equation

$$
\left[-\frac{d^{2}}{d \xi^{2}}-\frac{5}{2 \sigma^{10}}+\frac{15}{2} \xi^{2}-5 \sigma^{5} \mathrm{i} \xi^{3}+\mathcal{O}\left(\sigma^{6}\right)\right] \varphi^{[0]}(-\mathrm{i} \tau+\xi)=\sigma^{2} E_{n}^{[0]} \varphi^{[0]}(-\mathrm{i} \tau+\xi)
$$




\section{C.2 The conclusion: the spectrum is $N$-dependent}

Once we introduce $T=T_{j}(L)$ as a set of roots of the elementary algebraic equation

$$
2 L(L+1)=(2 N+1)^{2}(10 N+3) T^{10 N+5}
$$

we may abbreviate

$$
\tau=\tau^{(N)}=\left|\left(\frac{2 L(L+1)}{(2 N+1)^{2}(10 N+3)}\right)^{1 /(10 N+5)}\right|
$$

and specify all the solutions,

$$
T_{1}=-\mathrm{i} \tau, \quad T_{j}=T_{j-1} e^{2 \mathrm{i} \pi /(10 N+5)}, \quad j=2,3, \ldots, 10 N+5 .
$$

We shall only use here $T_{1}=-\mathrm{i} \tau$ giving the maximal size of the complex shift $\varepsilon=\tau$.

Theorem 2. In the tobogganic cases numbered by the winding number $N$ the low-lying imaginary-cubic spectrum is prescribed by the estimate

$$
E_{n}^{[N]}=-\frac{10 N+5}{2} \tau^{6 N+3}+\frac{2 n+1}{2 N+1} \sqrt{\frac{(10 N+3)(10 N+5)}{2}} \tau^{N+1 / 2}+\mathcal{O}\left(\tau^{-(6 N+3) / 4}\right) .
$$

Proof. The use of the standard methods gives

$$
V_{\text {eff }}(y)=-\frac{1}{2}(2 N+1)^{2}(10 N+5) \tau^{10 N+3}+\omega_{(N)}^{2} \tau^{10 N+1} \xi^{2}+\mu(N) \tau^{10 N} \xi^{3}+\mathcal{O}\left(\tau^{10 N-1}\right)
$$

with

$$
\omega_{(N)}=(2 N+1) \sqrt{\frac{(10 N+3)(10 N+5)}{2}} .
$$

The resulting approximate equation

$$
\begin{aligned}
& {\left[-\frac{d^{2}}{d \xi^{2}}+\omega_{(N)}^{2} \tau^{10 N+1} \xi^{2}+\mu(N) \tau^{10 N} \xi^{3}+\cdots\right] \psi_{n}(-\mathrm{i} \tau+\xi)} \\
& \quad=\left\{E_{n}^{[N]}(2 N+1)^{2}\left[\tau^{4 N}+\ldots\right]+\frac{1}{2}(2 N+1)^{2}(10 N+5) \tau^{10 N+3}\right\} \psi_{n}(-\mathrm{i} \tau+\xi)
\end{aligned}
$$

rescales in the form $\xi \rightarrow \sigma \xi$ using $\sigma=\tau^{-(10 N+1) / 4}$,

$$
\left[-\frac{d^{2}}{d \xi^{2}}-\frac{5}{2 \sigma^{10}}+\frac{15}{2} \xi^{2}-5 \sigma^{5} \mathrm{i} \xi^{3}+\mathcal{O}\left(\sigma^{6}\right)\right] \varphi^{[N]}(-\mathrm{i} \tau+\xi)=\sigma^{2} E_{n}^{[N]} \varphi^{[N]}(-\mathrm{i} \tau+\xi)
$$

and the closed formula for the energies follows.

In terms of a parameter $\rho=1 /(\ell+1 / 2)^{2}$ our approximate spectrum may be also rescaled giving the function $F_{n}^{(N)}=\rho^{3 / 5} E_{n}^{(N)}$ with the smooth and $\rho$-dependence which remains bounded even in the infinitely-large- $\ell$ limit. This is illustrated by Fig. 9 (units omitted as inessential).

Naturally, the scaling is unnecessary for the finite values of $\ell$. Our last Fig. 10 displays, therefore, the segments of the spectra of energies (with $n=0,1,2,3,4$ ) for the first four winding numbers $N=0,1,2$ and 3 . 


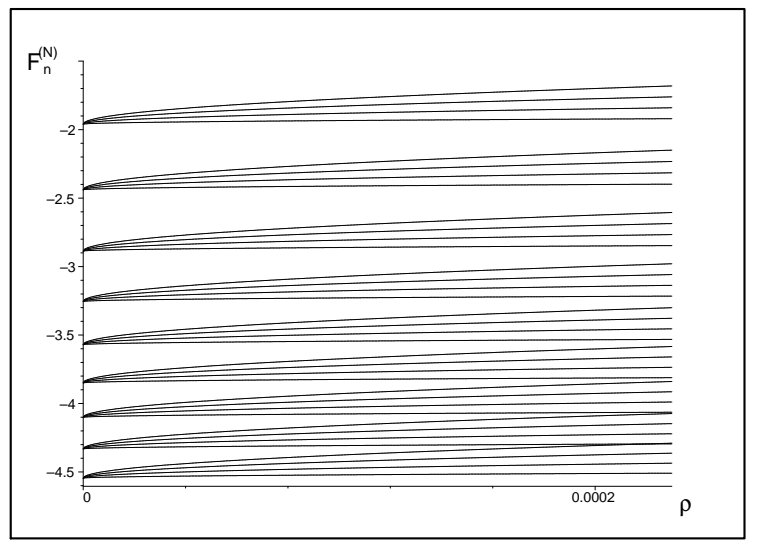

Figure 9. The rescaled tobogganic energies $F_{n}^{(N)}=\rho^{3 / 5} E_{n}^{(N)}(\rho)$ in the imaginary cubic well. The first four lowest energies with $n=0,1,2,3$ are sampled at very small $\rho=1 /(\ell+1 / 2)^{2}$. The increase of the winding $N=0,1, \ldots, 8$ pushes the spectrum downwards.

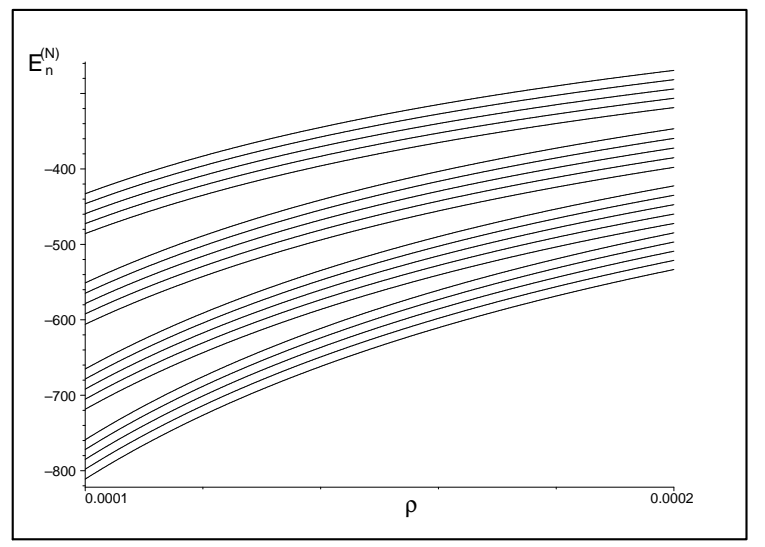

Figure 10. The unrescaled tobogganic energies $E_{n}^{(N)}(\rho)$ in the imaginary cubic well. The first five lowest energies with $n=0,1,2,3,4$ are sampled in a non-asymptotic interval of $\rho>\rho_{0}>0$. The result is shown for windings $N=0,1,2$ and 3 .

\section{Acknowledgements}

Work supported by MŠMT "Doppler Institute" project Nr. LC06002, by the GAČR grant Nr. P203/11/1433, by the Institutional Research Plan AV0Z10480505 and, last but not least, by the hospitality of STIAS in Stellenbosch in November 2010.

\section{References}

[1] Cooper F., Khare A., Sukhatme U., Supersymmetry and quantum mechanics, Phys. Rep. 251 (1995), 267385, hep-th/9405029.

[2] Jevicki A., Rodrigues J.P., Singular potentials and supersymmetry breaking, Phys. Lett. B 146 (1984), 55-58.

[3] Junker G., Supersymmetric methods in quantum and statistical physics, Text and Monographs in Physics, Springer-Verlag, Berlin, 1996.

Bagchi B.K., Supersymmetry in quantum and classical mechanics, Chapman \& Hall/CRC Monographs and Surveys in Pure and Applied Mathematics, Vol. 116, Chapman \& Hall/CRC, Boca Raton, FL, 2001.

[4] Das A., Pernice S.A., Supersymmetry and singular potentials, Nuclear Phys. B 561 (1999), 357-384, hep-th/9905135. 
[5] Buslaev V., Grecchi V., Equivalence of unstable anharmonic oscillators and double wells, J. Phys. A: Math. Gen. 26 (1993), 5541-5549.

Andrianov A.A., Ioffe M.V., Cannata F., Dedonder J.-P., SUSY quantum mechanics with complex superpotentials and real energy spectra, Internat. J. Modern Phys. A 14 (1999), 2675-2688, quant-ph/9806019. Cannata F., Junker G., Trost J., Schrödinger operators with complex potential but real spectrum, Phys. Lett. A 246 (1998), 219-226, quant-ph/9805085.

Bender C.M., Boettcher S., Meisinger P.M., PT-symmetric quantum mechanics, J. Math. Phys. 40 (1999), 2201-2229, quant-ph/9809072.

[6] Bender C.M., Boettcher S., Real spectra in non-Hermitian Hamiltonians having $\mathcal{P} \mathcal{T}$ symmetry, Phys. Rev. Lett. 80 (1998), 5243-5246, physics/9712001.

[7] Znojil M., Conservation of pseudo-norm in $\mathcal{P} \mathcal{T}$ symmetric quantum mechanics, math-ph/0104012.

[8] Mostafazadeh A., Pseudo-Hermiticity versus $\mathcal{P} \mathcal{T}$ Symmetry: the necessary condition for the reality of the spectrum of a non-Hermitian Hamiltonian, J. Math. Phys. 43 (2002) 205-214, math-ph/0107001.

Mostafazadeh A., Pseudo-Hermiticity versus $\mathcal{P} \mathcal{T}$-symmetry. II. A complete characterization of nonHermitian Hamiltonians with a real spectrum, J. Math. Phys. 43 (2002), 2814-2816, math-ph/0110016. Mostafazadeh A., Pseudo-Hermiticity versus $\mathcal{P} \mathcal{T}$-symmetry. III. Equivalence of pseudo-Hermiticity and the presence of antilinear symmetries, J. Math. Phys. 43 (2002), 3944-3951, math-ph/0203005.

[9] Bender C.M., Brody D.C., Jones H.F., Complex extension of quantum mechanics, Phys. Rev. Lett. 89 (2002), 270402, 4 pages, Erratum, Phys. Rev. Lett. 92 (2004), 119902, 1 page, quant-ph/0208076.

[10] Dorey P., Dunning C., Tateo R., Spectral equivalences, Bethe ansatz equations, and reality properties in $\mathcal{P}$ T-symmetric quantum mechanics, J. Phys. A: Math. Gen. 34 (2001), 5679-5704, hep-th/0103051.

[11] Znojil M., Comment on: "Supersymmetry and singular potentials" by Das and Pernice [Nuclear Phys. B 561 (1999), 357-384], Nuclear Phys. B 662 (2003), 554-562, hep-th/0209262.

[12] Dorey P., Dunning C., Tateo R., The ODE/IM correspondence, J. Phys. A: Math. Theor. 40 (2007), R205R283, hep-th/0703066.

[13] Bender C.M., Making sense of non-Hermitian Hamiltonians, Rep. Progr. Phys. 70 (2007), 947-1018, hep-th/0703096.

[14] Mostafazadeh A., Pseudo-Hermitian representation of quantum mechanics, Int. J. Geom. Methods Mod. Phys. 7 (2010), 1191-1306, arXiv:0810.5643.

[15] Znojil M., Three-Hilbert-space formulation of quantum mechanics, SIGMA 5 (2009), 001, 19 pages, arXiv:0901.0700.

[16] Znojil M., $\mathcal{P} \mathcal{T}$-symmetric regularizations in supersymmetric quantum mechanics, J. Phys. A: Math. Gen. 37 (2004), 10209-10222, hep-th/0404145.

[17] Znojil M., $\mathcal{P} \mathcal{T}$-symmetric quantum toboggans, Phys. Lett. A 342 (2005), 36-47, quant-ph/0502041.

[18] Fernández F.M., Guardiola R., Ros J., Znojil M., A family of complex potentials with real spectrum, J. Phys. A: Math. Gen. 32 (1999), 3105-3116, quant-ph/9812026.

Znojil M., Spiked potentials and quantum toboggans, J. Phys. A: Math. Gen. 39 (2006), 13325-13336, quant-ph/0606166.

Novotný J., http://demonstrations .wolfram.com/TheQuantumTobogganicPaths/.

[19] Znojil M., Jakubský V., Supersymmetric quantum mechanics living on topologically nontrivial Riemann surfaces, Pramana J. Phys. 73 (2009), 397-404, arXiv:0904.2294.

[20] Correa F., Jakubský V., Nieto L.M., Plyushchay M.S., Self-isospectrality, special supersymmetry, and their effect on the band structure, Phys. Rev. Lett. 101 (2008), 030403, 4 pages, arXiv:0801.1671.

Correa F., Jakubský V., Plyushchay M.S., Finite-gap systems, tri-supersymmetry and self-isospectrality, J. Phys. A: Math. Theor. 41 (2008), 485303, 35 pages, arXiv:0806.1614.

Siegl P., Supersymmetric quasi-Hermitian Hamiltonians with point interactions on a loop, J. Phys. A: Math. Theor. 41 (2008), 244025, 11 pages.

Jakubský V., Nieto L.M., Plyushchay M.S., Klein tunneling in carbon nanostructures: a free-particle dynamics in disguise, Phys. Rev. D 63 (2011), 047702, 4 pages, arXiv:1010.0569.

[21] Andrianov A.A., Cannata F., Sokolov A.V., Non-linear supersymmetry for non-Hermitian, nondiagonalizable Hamiltonians. I. General properties, Nuclear Phys. B 773 (2007), 107-136, math-ph/0610024.

[22] Znojil M., Cannata F., Bagchi B., Roychoudhury R., Supersymmetry without hermiticity within $\mathcal{P} \mathcal{T}$ symmetric quantum mechanics, Phys. Lett. B 483 (2000), 284-289, hep-th/0003277. 
[23] Znojil M., PT symmetrized SUSY quantum mechanics, Czechoslovak J. Phys. 51 (2001), 420-428, hep-ph/0101038.

Znojil M., $\mathcal{P} \mathcal{T}$-symmetry and supersymmetry, in GROUP 24: Physical and Mathematical Aspects of Symmetries (Paris, July 15-20, 2002), IOP Publishing, Bristol, 2003, 629-632, hep-th/0209062.

[24] Znojil M., Non-Hermitian SUSY and singular, $\mathcal{P} \mathcal{T}$-symmetrized oscillators, J. Phys. A: Math. Gen. 35 (2002), 2341-2352, hep-th/0201056.

[25] Levai G., Znojil M., The interplay of supersymmetry and $\mathcal{P} \mathcal{T}$ symmetry in quantum mechanics: a case study for the Scarf II potential, J. Phys. A: Math. Gen. 35 (2002), 8793-8804, quant-ph/0206013.

Sinha A., Roy P., Generation of exactly solvable non-Hermitian potentials with real energies, Czechoslovak J. Phys. 54 (2004), 129-138, quant-ph/0312089.

[26] Caliceti E., Graffi S., Maioli M., Perturbation theory of odd anharmonic oscillators, Comm. Math. Phys. 75 (1980), 51-66.

Sibuya Y., Global theory of second order linear differential equation with polynomial coefficient, North Holland, Amsterdam, 1975.

Fernández F.M., Guardiola R., Ros J., Znojil M., Strong-coupling expansions for the $\mathcal{P} \mathcal{T}$-symmetric oscillators $V(r)=a i x+b(i x)^{2}+c(i x)^{3}$, J. Phys. A: Math. Gen. 31 (1998), 10105-10112.

[27] Znojil M., $\mathcal{P} \mathcal{T}$-symmetric harmonic oscillators, Phys. Lett. A 259 (1999), 220-223.

[28] Znojil M., $\mathcal{P} \mathcal{T}$-symmetric square well, Phys. Lett. A 285 (2001), 7-10, quant-ph/0101131.

Quesne C., Bagchi B., Mallik S., Bíla H., Jakubský V., Znojil M., PT -supersymmetric partner of a shortrange square well, Czechoslovak J. Phys. 55 (2005), 1161-1166, quant-ph/0507246.

[29] Albeverio S., Fei S.-M., Kurasov P., Gauge fields, point interactions and few-body problems in one dimension, Rep. Math. Phys. 53 (2004), 363-370, quant-ph/0406158.

[30] Znojil M., Tater M., Complex Calogero model with real energies, J. Phys. A: Math. Gen. 34 (2001), 17931803, quant-ph/0010087.

Znojil M., Low-lying spectra in anharmonic three-body oscillators with a strong short-range repulsion, J. Phys. A: Math. Gen. 36 (2003), 9929-9941, quant-ph/0307239.

Fring A., Smith M., Antilinear deformations of Coxeter groups, an application to Calogero models, J. Phys. A: Math. Theor. 43 (2010), 325201, 28 pages, arXiv:1004.0916.

[31] Znojil M., Quantum knots, Phys. Lett. A 372 (2008), 3591-3596, arXiv:0802.1318.

[32] Znojil M., Quantum toboggans: models exhibiting a multisheeted $\mathcal{P} \mathcal{T}$ symmetry, J. Phys. Conf. Ser. 128 (2008), 012046, 12 pages, arXiv:0710.1485.

[33] Wessels G.J.C., A numerical and analytical investigation into non-Hermitian Hamiltonians, Master Thesis, University of Stellenbosch, 2008.

[34] Bíla H., Spectra of $\mathcal{P} \mathcal{T}$-symmetric Hamiltonians on tobogganic contours, Pramana J. Phys. 73 (2010), 307-314, arXiv:0905.1498.

[35] Dorey P., Millican-Slater A., Tateo R., Beyond the WKB approximation in $\mathcal{P} \mathcal{T}$-symmetric quantum mechanics, J. Phys. A: Math. Gen. 38 (2005), 1305-1331, hep-th/0410013.

[36] Znojil M., Quantum toboggans with two branch points, Phys. Lett. A 372 (2008), 584-590, arXiv:0708.0087.

[37] Znojil M., Classification of oscillators in the Hessenberg-matrix representation, J. Phys. A: Math. Gen. 27 (1994), 4945-4968.

[38] Znojil M., Siegl P., Levai G., Asymptotically vanishing $\mathcal{P} \mathcal{T}$-symmetric potentials and negative-mass Schrödinger equations, Phys. Lett. A 373 (2009), 1921-1924, arXiv:0903.5468.

[39] Scholtz F.G., Geyer H.B., Hahne F.J.W., Quasi-Hermitian operators in quantum mechanics and the variational principle, Ann. Physics 213 (1992), 74-101.

[40] Znojil M., Topology-controlled spectra of imaginary cubic oscillators in the large-L approach, Phys. Lett. A 374 (2010), 807-812, arXiv:0912.1176.

[41] Znojil M., Gemperle F., Mustafa O., Asymptotic solvability of an imaginary cubic oscillator with spikes, J. Phys. A: Math. Gen. 35 (2002), 5781-5793, hep-th/0205181.

[42] Znojil M., Identification of observables in quantum toboggans, J. Phys. A: Math. Theor. 41 (2008), 215304, 14 pages, arXiv:0803.0403.

Znojil M., Geyer H.B., Sturm-Schrödinger equations: formula for metric, Pramana J. Phys. 73 (2010), 299-306, arXiv:0904.2293.

[43] Znojil M., Re-establishing supersymmetry between harmonic oscillators in $D \neq 1$ dimensions, Rend. Circ. Mat. Palermo (2) Suppl. (2003), no. 71, 199-207, hep-th/0203252. 
[44] Dieudonné J., Quasi-Hermitian operators, in Proc. Internat. Sympos. Linear Spaces (Jerusalem, 1960), Pergamon, Oxford, 1961, 115-122.

Williams J.P., Operators similar to their adjoints, Proc. Amer. Math. Soc. 20 (1969), 121-123.

[45] Langer H., Tretter Ch., A Krein space approach to $\mathcal{P} \mathcal{T}$-symmetry, Czechoslovak J. Phys. 54 (2004), 11131120.

[46] Lévai G., Znojil M., Systematic search for $\mathcal{P} \mathcal{T}$-symmetric potentials with real energy spectra, J. Phys. A: Math. Gen. 33 (2000), 7165-7180.

[47] Günther U., Langer H., Tretter Ch., On the spectrum of the magnetohydrodynamic mean-field $\alpha^{2}$-dynamo operator, SIAM J. Math. Anal. 42 (2010), 1413-1447, arXiv:1004.0231.

Znojil M., Günther U., Dynamics of charged fluids and $1 / \ell$ perturbation expansions, J. Phys. A: Math. Theor. 40 (2007), 7375-7388, math-ph/0610055.

[48] Rüter C.E., Makris K.G., El-Ganainy R., Christodoulides D.N., Segev D.N., Kip D., Observation of paritytime symmetry in optics, Nature Phys. 6 (2010), 192-195.

Berry M.V., Optical lattices with $\mathcal{P} \mathcal{T}$ symmetry are not transparent, J. Phys. A: Math. Theor. 41 (2008), 244007, 7 pages.

Makris K.G., El-Ganainy R., Christodoulides D.N., Musslimani Z.H., Beam dynamics in $\mathcal{P} \mathcal{T}$ symmetric optical lattices, Phys. Rev. Lett. 100 (2008), 103904, 4 pages.

[49] Hilgevoord J., Time in quantum mechanics, Amer. J. Phys. 70 (2002), 301-306.

[50] Bender C.M., Turbiner A., Analytic continuation of eigenvalue problems, Phys. Lett. A 173 (1993), $442-446$.

[51] Mostafazadeh A., Metric operator in pseudo-Hermitian quantum mechanics and the imaginary cubic potential, J. Phys. A: Math. Gen. 39 (2006), 10171-10188, quant-ph/0508195.

[52] Jones H.F., Mateo J., Equivalent Hermitian Hamiltonian for the non-Hermitian $-x^{4}$ potential, Phys. Rev. D 73 (2006), 085002, 4 pages, quant-ph/0601188.

Bagchi B., Fring A., Minimal length in quantum mechanics and non-Hermitian Hamiltonian systems, Phys. Lett. A 373 (2009), 4307-4310, arXiv:0907.5354. 